\title{
Intrinsic transverse momentum and evolution in weighted spin asymmetries
}

\author{
Jian-Wei Qiu $\oplus^{1, *}$ Ted C. Rogers $\oplus^{1,2, \dagger}$ and Bowen Wang $\oplus^{3, \neq}$ \\ ${ }^{1}$ Jefferson Lab, 12000 Jefferson Avenue, Newport News, Virginia 23606, USA \\ ${ }^{2}$ Department of Physics, Old Dominion University, Norfolk, Virginia 23529, USA \\ ${ }^{3}$ Zhejiang Institute of Modern Physics, Department of Physics, Zhejiang University, \\ Hangzhou, Zhejiang 310027, China
}

(Received 7 May 2020; accepted 8 June 2020; published 25 June 2020)

\begin{abstract}
The transverse momentum-dependent (TMD) and collinear higher twist theoretical factorization frameworks are the most frequently used approaches to describe spin-dependent hard cross sections weighted by and integrated over transverse momentum. Of particular interest is the contribution from small transverse momentum associated with the target bound state. In phenomenological applications, this contribution is often investigated using transverse momentum weighted integrals that sharply regulate the large transverse momentum contribution, for example, with Gaussian parametrizations. Since the result is a kind of hybrid of TMD and collinear (inclusive) treatments, it is important to establish if and how the formalisms are related in applications to weighted integral observables. The suppression of a large transverse momentum tail, for example, can potentially affect the type of evolution that is applicable. We find that a naive version of a widely used identity relating the $k_{T}^{2}$-weighted and integrated Sivers TMD function to a renormalized twist-3 function has strongly ambiguous ultraviolet contributions, and that corrections to it are not necessarily perturbatively suppressed. We discuss the implications for applications, arguing in particular that the relevant evolution for transverse momentum weighted and integrated cross sections with sharp effective large transverse momentum cutoffs is of the TMD form rather than the standard renormalization group evolution of collinear correlation functions.
\end{abstract}

DOI: 10.1103/PhysRevD.101.116017

\section{INTRODUCTION}

Understanding fully the single transverse-spin asymmetries (SSAs) of high energy scattering cross sections with the momentum transfer $Q \gg \Lambda_{\mathrm{QCD}}$ is still one of the most fascinating and challenging subjects in QCD since its discovery in hadronic $\Lambda^{0}$ production over 40 years ago [1]. The transverse SSA, defined as $A_{N}=\left(\sigma\left(S_{T}\right)-\right.$ $\left.\sigma\left(-S_{T}\right)\right) /\left(\sigma\left(S_{T}\right)+\sigma\left(-S_{T}\right)\right)$, has been observed in many cross sections $\sigma\left(S_{T}\right)$, involving a single transverse hadronic spin $S_{T}$, and can be as large as $30 \%-40 \%$ in the forward region of hadronic single pion production [2-4]. This contradicted expectations about the size of the asymmetry that were based on early theoretical calculations [5]. With the parity and time-reversal invariance of QCD, it was recognized that the nonvanishing $A_{N}$ is a consequence of

\footnotetext{
*jqiu@jlab.org

trogers@odu.edu

¥0617626@zju.edu.cn
}

Published by the American Physical Society under the terms of the Creative Commons Attribution 4.0 International license. Further distribution of this work must maintain attribution to the author(s) and the published article's title, journal citation, and DOI. Funded by SCOAP. nonperturbative partonic motion and its correlation with the direction of the observed hadronic spin. Thus, $A_{N}$ is a uniquely useful observable for probing a hadron's internal partonic structure and for studying quantum correlations between the partonic dynamics and emergent hadronic properties such as total spin [6].

The ability to understand $A_{N}$ in terms of the correlations between the partonic motion and hadronic spin relies on QCD factorization [7] since any cross section with an identified hadron (and any corresponding asymmetry) is not perturbatively calculable in QCD. A QCD factorization formalism for $A_{N}$ depends on the kinematics of measured cross section $\sigma\left(S_{T}\right)$. As a typical two-scale observable, for example, Drell-Yan lepton pair production by hard quarkantiquark annihilation, as shown in Fig. 1 for partonic targets, can have very different factorization formalisms for $\Delta \sigma\left(Q_{T}, Q ; S_{T}\right)=\sigma\left(Q_{T}, Q ; S_{T}\right)-\sigma\left(Q_{T}, Q ;-S_{T}\right)$, defined as the difference between cross sections with the transverse spin flipped. In addition, it has two observed momentum scales associated with the virtual photon: its invariant mass $Q=\sqrt{q^{2}} \gg \Lambda_{\mathrm{QCD}}$ and the transverse momentum $Q_{T}$ with respect to the collision axis of $p$ and $p^{\prime}$. When the active parton's transverse momentum is in the range of $k_{T} \sim Q_{T} \ll Q$, represented by Fig. 1(a), intrinsic transverse momentum can be important and transverse 


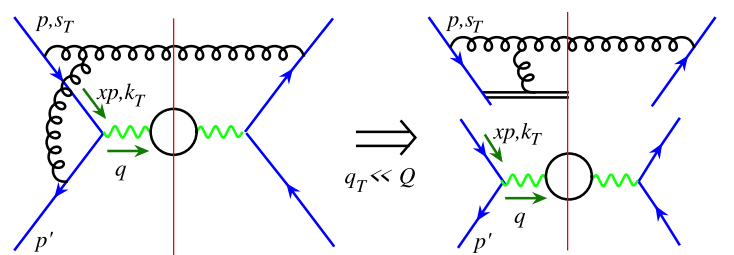

(a)

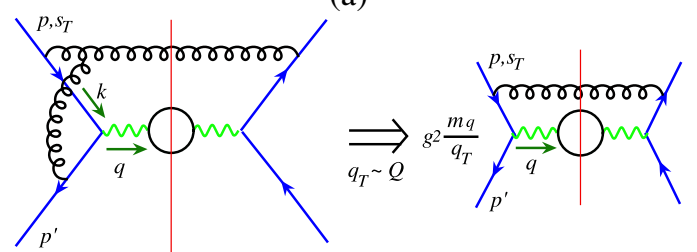

(b)

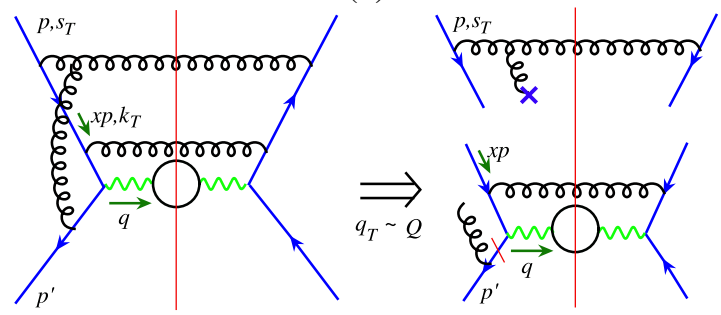

(c)

FIG. 1. Low-order diagrams for $A_{N}$ of Drell-Yan lepton pair production by hard quark-antiquark annihilation: (a) TMD factorization when $k_{T} \sim Q_{T} \ll Q=\sqrt{q^{2}}$. (b) Fixed-order perturbative QCD (pQCD) calculation when $Q_{T} \sim Q$. (c) Twist-3 collinear factorization when $k_{T} \ll Q_{T} \sim Q$.

momentum-dependent (TMD) factorization is relevant. Then the contribution to $A_{N}$ from the diagram on the left can be approximately represented (schematically) by the TMD factorized expression on the right,

$$
\begin{aligned}
& \Delta \sigma\left(Q_{T}, Q ; S_{T}\right) \\
& \quad \propto \hat{\sigma}_{q \bar{q} \rightarrow \bar{l}(Q)}^{(0)} \otimes f_{\bar{q} / \bar{q}}^{(0), \mathrm{TMD}} \otimes k_{\mathrm{T}} f_{1 T, q / q}^{\perp(1)}+O\left(Q_{T} / Q\right),
\end{aligned}
$$

where $\hat{\sigma}^{(0)}$ is the lowest-order partonic Drell-Yan cross section, shown as the lower factorized diagram on the right of the arrow in Fig. 1(a), $f_{\bar{q} / \bar{q}}^{(0), \text { TMD }}\left(x^{\prime}, k_{T}^{\prime}\right)$ is the zeroth-order unpolarized TMD antiquark distribution of an antiquark, which is proportional to $\delta\left(1-x^{\prime}\right) \delta^{2}\left(k_{T}^{\prime}\right)$ in lowest-order perturbation theory, $f_{1 T, q / q}^{\perp(1)}\left(x, k_{T}\right)$ is the first-order quark Sivers TMD function of a quark, given by the top diagram on the right of the arrow in Fig. 1 , and $\otimes$ indicates the convolution of the active parton's momentum, both longitudinal and transverse in this case. The asymmetry $A_{N}$ is generated by the nonvanishing Sivers function [8-10].

But, when $Q_{T} \sim Q$, the same diagram, now symbolized by Fig. 1(b), would give a leading fixed-order contribution to $A_{N}$ while the loop on the left generates the needed phase and the quark mass $m_{q}$ generates the spin flip for the $A_{N}$. This leads to an asymmetry proportional to $g_{s}^{2} m_{q} / Q_{T}$ with strong coupling constant $g_{s}$, which was predicted to be very small in view of $m_{q} \ll Q_{T} \sim Q$ [5].

At the same $Q_{T} \sim Q$, however, additional mechanisms can generate transverse SSAs and these are symbolized in Fig. 1(c). When the transverse momentum of the active parton for the hard scattering to produce the lepton pair is in the range $k_{T} \ll Q_{T} \sim Q$, the formally higher-order diagram on the left can be factorized in terms of twist-3 collinear factorization

$$
\begin{aligned}
& \Delta \sigma\left(Q_{T}, Q ; S_{T}\right) \\
& \quad \propto \hat{H}_{q(g) \bar{q} \rightarrow \bar{l}(q)}^{(1)} \otimes f_{\bar{q} / \bar{q}}^{(0) \text { coll }} \otimes T_{q(g) / q}^{(1)}+O\left(\Lambda_{\mathrm{QCD}} / Q\right),
\end{aligned}
$$

where $\hat{H}^{(1)}$ is the lowest-order partonic hard part to produce the SSA of high- $Q_{T}$ lepton pair production. This is shown as the bottom diagram on the right of the arrow in Fig. 1(c), with the unpinched pole of the antiquark line having a (red) bar to indicate the needed phase. The active quark-gluon composite state allows for the helicity to flip between the left and the right of the cut in this diagram, even with zero quark mass. The $f_{\bar{q} / \bar{q}}^{(0) \text { coll }}\left(x^{\prime}\right)$ is the zeroth-order unpolarized twist-2 collinear antiquark distribution of an antiquark at lowest order of perturbation theory, which is proportional to $\delta\left(1-x^{\prime}\right), T_{q(g) / q}^{(1)}(x)$ is the first-order twist-3 quark-gluon correlation function of a quark, given by the top diagram on the right, and $\otimes$ indicates the convolution of active parton's longitudinal momentum fractions. The typical transverse momenta of active partons here, which are expected to be much smaller than the hard scale, $Q_{T} \sim Q$, are integrated into the twist-3 quark-gluon correlation function, whose size is determined by the imbalance of quark motion generated by the color Lorentz force (the gluon) in defining the twist-3 quark-gluon correlation functions [11-14].

Both TMD and twist-3 collinear factorization formalisms, in Eqs. (1) and (2), respectively, have been argued to be valid to all orders in QCD perturbation theory for their respective kinematical regimes [15-20]. In an overlap region where $\Lambda_{\mathrm{QCD}} \ll Q_{T} \ll Q$, the TMD and twist-3 collinear factorization formalisms for the SSAs were shown to be consistent with each other [21-23] when the active parton $k_{T}$ and the phase of the Sivers TMD function are perturbatively generated by the twist- 3 mechanism.

Both TMD and twist-3 collinear factorization approaches have also been used frequently to describe the transverse moment of two-scale spin-dependent hard cross sections and their asymmetries, by integrating over transverse momentum $Q_{T}$ while weighting by a single power of $\mathbf{Q}_{T}$, leaving the observables with only a single large momentum transfer $Q$ [24-28]. In principle, the moments (or the asymmetries of the moments) of $Q_{T}$ distributions should be described by a QCD collinear factorization formalism, if one exists, since the active parton's $k_{T}$ should be much less than the single hard momentum transfer $Q$. 
In practice, however, both factorization approaches have been adopted for evaluating the moments of the $Q_{T}$ distributions. For example, an unpolarized Drell-Yan-like cross section $\frac{d \sigma}{d Q^{2}}$ is often calculated in terms of QCD collinear factorization with perturbatively calculated hard parts $\frac{d \hat{\sigma}_{i j}}{d Q^{2}}$ convoluted with two twist-2 collinear parton distribution functions (PDFs) $f_{i / H}(x)$ [7]. (Here we suppress the factorization scale and active parton flavor indexes, $i, j=q, \bar{q}, g$.) The same observable can be viewed as the zeroth moment of the $Q_{T}$ distribution, $\frac{d \sigma}{d Q^{2}}=$ $\int d Q_{T}^{2}\left(Q_{T}^{2}\right)^{0} \frac{d \sigma}{d Q^{2} d Q_{T}^{2}}$, with the $\frac{d \sigma}{d Q^{2} d Q_{T}^{2}}$ evaluated in terms of the TMD factorization formalism and unpolarized TMD PDFs $f_{i / H}\left(x, k_{T}\right)$ when $k_{T} \sim Q_{T} \ll Q$, along with a proper matching when $Q_{T}$ becomes larger $\left(Q_{T} \sim Q\right)$ to a cross section calculation $\frac{d \sigma^{\text {Pert }}}{d Q^{2} d Q_{T}^{2}}$ performed in terms of QCD collinear factorization with twist-2 collinear PDFs [15]. Both approaches are well-defined within QCD perturbation theory and within the frameworks of their corresponding factorization theorems.

Of course, the above remarks apply similarly to other processes with a transversely polarized hadronic target, particularly semi-inclusive deep inelastic scattering (SIDIS) with its typically smaller $Q$ and higher sensitivity to nonperturbative hadronic structure.

A commonly used relation between TMD PDFs and twist-2 collinear PDFs,

$$
\int \mathrm{d}^{2} \boldsymbol{k}_{\mathrm{T}} f_{i / H}\left(x, k_{\mathrm{T}}\right)=f_{i / H}(x),
$$

connects the two approaches to each other, up to $O\left(\alpha_{s}\right)$ suppressed terms associated with different ways of including high-order corrections [29]. When the full TMD factorization formalism is used for the region of $Q_{T} \ll Q$, and optimized for the region $\Lambda_{\mathrm{QCD}} \ll Q_{T} \ll Q$ with resummed $\ln \left(Q^{2} / Q_{T}^{2}\right)$-enhanced effects taken into account, the cross section as a zeroth moment receives corrections to Eq. (3), as demonstrated for inclusive Higgs production in a Drell-Yan-like process [29].

It has been proposed that the TMD and twist- 3 collinear factorization approaches to describing the transverse moment of the two-scale spin-dependent hard cross sections and their asymmetries are connected through a wellknown relation between the Sivers TMD function $f_{1 T, q / H}^{\perp}\left(x, k_{\mathrm{T}}\right)$ of hadron $H$ and the twist-3 quark-gluon correlation function $T_{q(g) / H}(x)$ [30],

$$
\int \mathrm{d}^{2} \boldsymbol{k}_{\mathrm{T}} \frac{k_{\mathrm{T}}^{2}}{M^{2}} f_{1 T, q / H}^{\perp}\left(x, k_{\mathrm{T}}\right)=-\frac{1}{M} T_{q(g) / H}(x),
$$

in an analog to the relation in Eq. (3), where factors of the hadron mass (labeled $M$ ) are included by convention to make both sides dimensionless. To simplify notation, we have dropped the usual second argument of the twist-3 quark-gluon correlation function $T_{q(g) / H}(x, x)$ since for our purposes we will only be interested in the case where both active quark momentum fractions are equal. For the relative minus sign in Eq. (4), the Wilson line in the Sivers TMD function should be understood to point in the direction relevant to lepton-hadron SIDIS [31], which would require an extra minus sign in Eq. (4) if we prefer to use the Sivers TMD function extracted from the Drell-Yan-type processes. In this paper, we try to verify the relation in Eq. (4) and to understand how it is similar or different from the unpolarized analog in Eq. (3).

The moment of the Sivers function on the left side of Eq. (4) arises naturally in studies of the moment or weighted transverse SSAs. For example, the TMD factorized expression in Eq. (1) can be used to evaluate the $\mathbf{Q}_{T}$-weighted asymmetry if one assumes it is approximately valid for the full range of $\mathbf{Q}_{T}$-integration, that is, if one neglects the $Q_{T} \sim$ $Q$ "Y-term" correction and assumes exact validity for Eq. (4). This results in a factorized expression proportional to the integral on the left side of Eq. (4) [30].

The equality in Eq. (4) is widely understood to imply that $T_{q(g) / H}(x)$ and $f_{1 T, q / H}^{\perp}\left(x, k_{\mathrm{T}}\right)$ are essentially different ways of representing similar physics [32-34], namely that of intrinsic nonperturbative parton transverse momentum inside a hadron target around $k_{T} \sim \Lambda_{\mathrm{QCD}}$. This view has motivated various interpretations of experimental data, including, for example, suggestions of tension in the phenomenology of the Sivers effect [31,32]. Equation (4) is also a common ingredient in phenomenological applications of twist-3 factorization because practical functional representations of the twist-3 quark-gluon correlation function are obtained via Eq. (4) from phenomenological extractions of the Sivers function [35]. It has also been suggested that Eq. (4) provides a kind of loophole around the problems with TMD factorization that arise in certain processes [36].

In Eq. (4), both the Sivers TMD function $f_{1 T, i / H}^{\perp}\left(x, k_{\mathrm{T}}\right)$ and the twist-3 correlation function $T_{q(g) / H}(x)$ are nonperturbative but could in principle be extracted from physically measured SSAs. If the $\mathrm{d}^{2} \boldsymbol{k}_{\mathrm{T}}$-integration of a measured $f_{1 T, i / H}^{\perp}\left(x, k_{\mathrm{T}}\right)$ weighted by $k_{\mathrm{T}}^{2}$ converges, then the relation in Eq. (4) can be tested for its $Q^{2}$ dependence as well as its $x$ dependence. However, the relation in Eq. (4) is often used in the literature as an identity to replace one side by the other side to help in the extraction of the Sivers TMD functions (or twist-3 correlation functions) and thus does not treat them as two different functions. Therefore, the precise reliability of the relation in Eq. (4) can impact ongoing community efforts to extract nonperturbative TMD correlation functions and to explore hadron's internal partonic structure and its correlation to the emergent hadronic properties.

In phenomenological applications, an ambiguity immediately arises as to what type of $Q^{2}$ dependence or scale 
evolution should be expected for the weighted integral on the left side of Eq. (4) [37]. Taken literally, the right side of the equation implies that the $Q^{2}$ dependence should follow from a Dokshitzer-Gribov-Lipatov-Altarelli-Parisi (DGLAP)-type evolution of twist-3 quark-gluon correlation functions $[38,39]$ since $T_{q(g) / H}(x)$ should be extracted from the observed $A_{N}$ factorized in terms of the twist-3 collinear factorization. By contrast, the $f_{1 T, i / H}^{\perp}\left(x, k_{\mathrm{T}}\right)$ is to be extracted from the observed $A_{N}$ differential in transverse momentum and factorized in terms of TMD factorization, whose $Q^{2}$ dependence should follow the Collins-Soper style of evolution $[15,40]$, and without a full treatment of the large $\mathbf{Q}_{T} \sim Q$ tail the additional transverse momentum integral would not change this $Q^{2}$ dependence to the DGLAP type.

Like all QCD factorization formalisms, both the TMD and twist-3 collinear factorization theorems for SSAs are constructed such that collinear and infrared (IR) sensitivity is automatically removed from the partonic scattering process and placed in the nonperturbative long-distance but universal TMD functions and twist-3 quark-gluon correlation functions, respectively. The predictive power of the TMD and twist-3 collinear factorization in Eqs. (1) and (2) relies on (a) the universality of the Sivers TMD functions and twist-3 collinear quark-gluon correlation functions and, by extension, (b) their abilities to systematically remove the collinear and infrared sensitivities of the corresponding partonic scattering to ensure the infrared safety of $\hat{\sigma}$ in Eq. (1) and $\hat{H}$ in Eq. (2) order-by-order in QCD perturbation theory at all applicable momentum scales. Given the difference in operators defining the Sivers TMD function and the twist-3 quark-gluon correlation function, it is not immediately clear that one should expect $T_{q(g) / H}(x)$ and $f_{1 T, q / H}^{\perp}\left(x, k_{\mathrm{T}}\right)$ to have comparable nonperturbative small transverse momentum behavior, since the partonic versions of such objects and their scale evolution are clearly qualitatively different beyond the tree level $[38,41]$. The question is whether a weighted $k_{T^{-}}$ integration of $f_{1 T, q / H}^{\perp}\left(x, k_{\mathrm{T}}\right)$ like Eq. (4) would make them be the same.

Furthermore, in order to apply QCD factorization to the moment of spin-dependent hard cross sections and their asymmetries beyond the tree level in perturbative calculations, the operators that define Sivers TMD functions and twist-3 correlation functions in Eq. (4) should be the renormalized ones, and the renormalization of corresponding nonlocal operators needs to be specified. Otherwise, the derivation of Eq. (4) involves manipulations with infinite quantities [30]. So, in view of the widespread use of Eq. (4) it is important to characterize possible violations to it that might become apparent once the divergent behavior is taken into account. Indeed, the violation of Eq. (4) as an exact statement is already well known (see, for example, Ref. [42], along with the discussion there regarding sensitivity to large $k_{T}$ cutoff schemes). In particular, the removal of ultraviolet (UV) regulators does not generally commute with the evaluation of transverse momentum integrals. However, it is typically assumed that, after $k_{T}$-cutoffs are in place, violations to Eq. (4) correspond to small perturbative corrections and that it can be viewed as a kind of zeroth-order approximation.

There are a number of open questions in the treatment of factorization for weighted inclusive observables generally, and we do not intend to address them all here. Indeed, with only one large momentum scale observed, a complete derivation of collinear factorization for fully inclusive weighted moments in terms of twist-3 functions alone does not yet, to our knowledge, exist. Instead, we will highlight particular issues that arise by focusing on the properties of individual parton correlation functions when their definitions are taken literally. Nevertheless, we emphasize that, for implementations that focus on the small or nonperturbative transverse momentum region while suppressing the large transverse momentum tail, factorization with TMD correlation functions is natural.

Within the assumption that all operator matrix elements are calculated using standard renormalization, we will argue using an explicit calculation that the breakdown of Eq. (4) is not perturbatively suppressed in the normal sense and is sensitive instead to a collinear regulator. We propose, therefore, to take Eq. (4) as a definition for the UV behavior of $T_{q(g) / H}(x)$ rather than as a derived result, at least for those observables that focus on the small transverse momentum region. Moreover, if transverse momentum cutoffs are sharp enough to retain sensitivity to nonperturbative intrinsic transverse momentum, as with, for example, narrow Gaussian parametrizations, then evolution of the corresponding weighted and integrated asymmetries should be for TMD functions rather than through collinear evolution. The Gaussian (or similar) ansatz approach to TMD phenomenology has met with significant success in applications [43-45] and is an approach that maintains a more natural link to intrinsic nonperturbative physics than those that focus more on accurately describing a broad perturbative transverse momentum tail.

Similar identities to Eq. (4) are used to relate other kinds of twist-3 collinear and TMD functions, for example, the Collins fragmentation function [46-48], and there are many similar proposed relations between twist-3 and TMD correlation functions [e.g., Eqs. (C13)-(C15) of [49] ]. Thus, our results potentially impact the study of weighted-integrated correlation functions more broadly.

The rest of this paper is organized as follows: In the next section, we introduce our conventions for the renormalization of PDFs and, in general, parton correlation functions. As an example, and to set up later discussions of Eq. (4), in Sec. III we further discuss the relation in Eq. (3) relating spin averaged TMD PDFs and collinear PDFs. In Sec. IV, we specify how the proposed identity in Eq. (4) is to be 
tested and show the violation of the identity in terms of an explicit lowest-order calculation in perturbative QCD in Sec. V. Finally, we discuss our results and our proposal for the treatment of the evolution of weighted asymmetries in Sec. VI.

\section{RENORMALIZATION}

We will refer to the renormalization of PDFs in the standard sense of a renormalization of a generalized operator product. So, for example, the renormalized collinear PDF for a quark in a hadron is

$$
f_{i / H}(x ; \mu)=Z_{i j} \otimes f_{j / H, 0},
$$

where $i, j$ represent the quark flavor. The bare PDF $f_{j / H, 0}(x)$ has the usual definition of a PDF, but defined with bare fields. The $\otimes$ denotes the usual convolution products over longitudinal momentum fractions that appear in collinear factorization, and $\mu$ is the usual renormalization group scale. Our calculations that use dimensional regularization will work in dimension $D=4-2 \epsilon$ and use a generalized minimal subtraction scheme for renormalization, in which case the $Z_{i j}$ beyond zeroth order consist only of $\epsilon$-poles with mass-independent coefficients.

It is important to note that for higher twist operators renormalization can mix with operators of lower dimension.

Renormalization is not the only valid way to define the ultraviolet behavior of collinear correlation functions, but it comes with many desirable features, including the automatic cancellation of light cone divergences and order-byorder validity of number and momentum sum rules. We therefore view it as the default approach.

Renormalization works similarly for TMD PDFs, though an extra kind of generalized renormalization is needed in association with light cone divergences [16]. Such issues will not arise directly in this paper, however.

For the message of this paper to be clear, it is important to recall that with the renormalization approach to PDFs, virtual and real UV divergences need to be consistently regulated in the same way-see Sec. VI below for more on this.

\section{COMPARISON WITH UNPOLARIZED CASE}

The equality in Eq. (3) relating unpolarized TMD PDFs and collinear PDFs is similar to the relation in Eq. (4) in the sense that a moment of the TMD PDF is related to a corresponding collinear PDF. But the two equalities in Eqs. (3) and (4) are also fundamentally different in the nature of the operators involved.

For the relation in Eq. (3), the nonlocal operators defining the TMD PDFs on the left-hand side and the nonlocal operators of corresponding collinear PDFs on the right-hand side share the same leading twist local operators when the operator product expansion is applied to these nonlocal operators. That is, both sides of the relation in Eq. (3) share the same leading twist, leading-order perturbative collinear, and UV behavior. As discussed earlier, the same collinear sensitivities in perturbative calculations from the two sides of the equality is a requirement for factorization if both sides of Eq. (3) are to be equally valid definitions for the collinear PDF. The integration over $k_{T}^{2}$, specifically the transverse momentum flow between the active quark in the scattering amplitude and its complex conjugate, picks up the leading twist operators with no transverse separation, which are logarithmically UV divergent and require renormalization. Consequently, differences between the two sides of Eq. (3) could potentially include the effect of different choices (or schemes) for renormalizing the perturbative leading twist UV divergence and how this differs from the UV regulator of the integration over the active parton's transverse momentum $k_{T}^{2}$. This leading twist scheme dependence does not change the collinear sensitivities of either side in Eq. (3). That is, any possible difference between the two sides of the relation in Eq. (3) is infrared insensitive or perturbatively calculable. Before turning to the extra complications that might arise with Eq. (4), we quantify the relation in Eq. (3) in the rest of this section.

As is well known, Eq. (3) is actually valid up to perturbative corrections for renormalized PDFs (both collinear and TMD) when a cutoff $k_{c}$ is imposed on the transverse momentum integral and if TMD PDFs are defined in any of the usual senses [16,41,50-61] that are currently used. We may state this explicitly by first defining

$$
\Delta f\left(k_{c}\right) \equiv \pi \int_{0}^{k_{c}^{2}} \mathrm{~d} k_{\mathrm{T}}^{2} f_{i / H}\left(x, k_{\mathrm{T}} ; \mu\right)-f_{i / H}(x ; \mu),
$$

where the definition of $f_{i / H}\left(x, k_{\mathrm{T}} ; \mu\right)$ is any of the standard TMD definitions, and $f_{i / H}(x ; \mu)$ is the standard renormalized parton density. Then it is straightforward to verify that the following factorization holds:

$$
\begin{aligned}
\Delta f\left(k_{c}\right)= & \sum_{i j} \mathcal{C}_{i j}\left(x / x^{\prime}, \alpha_{s}(\mu), L\right) \otimes f_{j / H}(x ; \mu) \\
& +O\left(\frac{\Lambda_{\mathrm{QCD}}^{2}}{k_{c}^{2}}\right)
\end{aligned}
$$

where the $\mathcal{C}_{i j}$ are mass-independent generalized functions that depend on $\mu$ only through $\alpha_{s}(\mu)$ and powers of the logarithm

$$
L\left(k_{c} / \mu\right) \equiv \ln \left(\frac{\mu^{2}}{k_{c}^{2}}\right)
$$

The $\mathcal{C}_{i j}$ start at order $\alpha_{s}(\mu)$ or higher. Therefore, as long as the cutoff $k_{c}$ is fixed roughly at order $\mu$, corrections 
to Eq. (3) are suppressed by at least a power of $\alpha_{s}(\mu)$. When both the $\alpha_{s}\left(k_{c}\right)$ - and $\left(\Lambda_{\mathrm{QCD}}^{2} / k_{c}^{2}\right)$-suppressed terms in Eq. (7) are dropped, the identity in Eq. (3) is restored. Verifying the above is possible to do directly in renormalizable model field theories or in pQCD order-by-order.

The purpose of the discussion above is to make statements about relations like Eqs. (3) or (4) holding at "lowest-order" precise. Then in the next two sections we explain why a statement analogous to Eq. (7) fails for Eq. (4) if applied to ordinary renormalized correlation functions.

\section{SPIN-DEPENDENT CASE}

Now we return to Eq. (4). The general form of the operator definition of the (pole part of the) twist-3 quarkgluon correlation function is

$$
\begin{aligned}
T_{i(g) / H}(x)= & g_{s} \epsilon^{S_{T} \alpha} g_{\alpha \beta} \int \frac{\mathrm{d} \xi^{-} \mathrm{d} \eta^{-}}{4 \pi} e^{i x P^{+} \xi^{-}}\langle P, S| \bar{\psi}_{i}(0) G^{\beta+} \\
& \times\left(\eta^{-}\right) \gamma^{+} \psi_{i}\left(\xi^{-}\right)|P, S\rangle .
\end{aligned}
$$

$S_{T}$ is the transverse spin of the target and $G^{\mu \nu}$ is the gluonic field strength tensor. The analog of Eq. (5) is a renormalized twist-3 quark-gluon correlation function

$$
\begin{aligned}
T_{i(g) / H}(x ; \mu)= & \sum_{i j} Z_{a, i j} \otimes T_{j(g) / H, 0} \\
& +\sum_{b, i j} m_{b, i j} Z_{b, i j} \otimes h_{j / H, 0, b} .
\end{aligned}
$$

The $T_{i(g) / H, 0}$ are defined as in Eq. (9), but here specifically with bare fields, the $h_{i / H, 0, b}$ are any of the possible lower twist bare collinear operator matrix elements that might be necessary in the renormalization, and the $m_{b, i j}$ are the renormalized masses of any of the fields. As before, $i$ and $j$ are parton flavor indices. The $Z_{a}$ and $Z_{b}$ coefficients are renormalization factors respectively for the bare collinear twist-3 function $T_{j(g) / H, 0}$ and any other lower dimension operators. In dimensional regularization with generalized minimal subtraction, they are massindependent poles in $\epsilon$.

The analog of Eq. (6) for Eq. (4) is

$$
\begin{aligned}
\Delta f_{1 T}^{\perp}\left(k_{c}\right) \equiv & \pi \int_{0}^{k_{c}^{2}} \mathrm{~d} k_{\mathrm{T}}^{2} \frac{k_{\mathrm{T}}^{2}}{M^{2}} f_{1 T, i / H}^{\perp}\left(x, k_{\mathrm{T}} ; \mu\right) \\
& +\frac{1}{M} T_{i(g) / H}(x ; \mu) .
\end{aligned}
$$

If a version of Eq. (4) held at zeroth order, then it would have to be possible to express $\Delta f_{1 T}^{\perp}\left(k_{c}\right)$ in the following factorized way:

$$
\begin{aligned}
M \Delta f_{1 T}^{\perp}\left(k_{c}\right) \stackrel{? ?}{=} & \sum_{i j} \mathcal{C}_{i j}\left(x / x^{\prime}, L, \alpha_{s}(\mu)\right) \otimes T_{j(g) / H}(x ; \mu) \\
& +\sum_{b, i j} m_{b, i j} \mathcal{C}_{b, i j}\left(x / x^{\prime}, L, \alpha_{s}(\mu)\right) \otimes h_{j / H, b}(x ; \mu) \\
& +O\left(\frac{\Lambda_{\mathrm{QCD}}^{2}}{k_{c}^{2}}\right)
\end{aligned}
$$

analogously to the unpolarized case in Eq. (7), but now allowing for mixing with lower dimensional operators. Similar to Eq. (7), if Eq. (4) is valid up to perturbative corrections, then the collinear matrix elements on the right side of Eq. (12) must be operators with equal or lower dimension to $T_{i(g) / H}(x ; \mu)$, and the $\mathcal{C}_{i j}$ must begin at order $\alpha_{s}$ or higher and involve only the logarithms $L$ [Eq. (8)]. The "??" is to emphasize that Eq. (12) is provisional and will actually turn out not to hold.

\section{NONVERIFICATION}

A complication with checking relations like Eq. (12) directly is that the functions involved are nonperturbative. However, the generic behavior that we are interested in testing can be checked order-by-order in any theory with the relevant properties of renormalizability and a gauge interaction, for example, in a model field theory with a spectator. Moreover, if the factorization in Eq. (12) were true generally, then it must hold order-by-order for partonic targets. We consider, therefore, a nonzero mass quark in pQCD as the target to remain as close to true QCD as possible, while the quark mass $m_{q}$ also serves as a regulator for perturbative collinear singularities. The lowest-order nonvanishing graphs are shown in Fig. 2, with Fig. 2(a) representing the twist- 3 collinear calculation [the second term on the right side of Eq. (11)] and Fig. 2(b) representing the TMD PDF calculation [the integrand for the first term on the right side of Eq. (11)]. ${ }^{1}$ Although we are calculating in perturbation theory, the calculation must be viewed as a kind of model since the functions are nonperturbative, and we must assume that a suitable infrared regulator has been imposed on higher-order graphs, though we will not need to make the specific regulator explicit here because all the graphs in Fig. 2 are infrared and collinear finite with a nonzero quark mass and a fixed momentum fraction $x$.

Both calculations in Fig. 2 proceed similarly, up to the factor of $k_{T}^{2} / M^{2}$ and the absent integral over transverse momentum for the TMD PDF case. Fortunately, several features of Figs. 2(a) and 2(b) simplify their calculation. First, the TMD PDF case is finite in both the UV and IR, even with a zero mass gluon. Second, if we restrict to the class of nonsingular covariant gauges, they are gauge

\footnotetext{
${ }^{1}$ We have labeled the exchanged gluon momentum by $q$ to be consistent with the notation in [42]. This should not be confused, however, with a virtual photon momentum like the one in Fig. 1.
} 


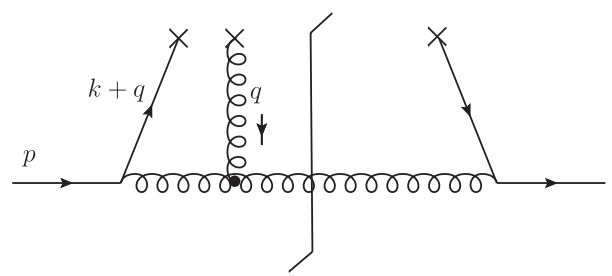

(a)

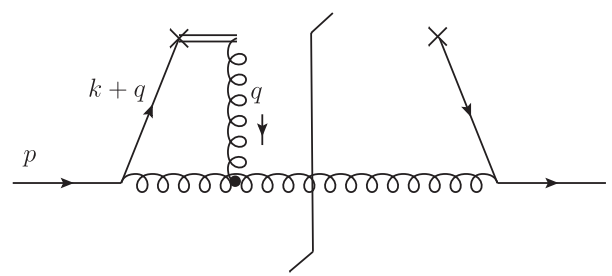

(b)

FIG. 2. (a) Lowest-order pole part of the twist-3 quark-gluon correlation function. (b) Lowest-order TMD Sivers function. The calculations are nearly identical up to the overall $-1 / 2 M$, the factor of $k_{T}^{2}$, and the integral over transverse momentum in the case of the twist-3 quark-gluon correlation function.

independent as can be seen from the fact they (and their Hermitian conjugates) are the only graphs that contribute at $O\left(\alpha_{s}\right)$ to the transverse single spin asymmetry with unpolarized active quark, so no subtleties associated with the Wilson line in the twist-3 quark-gluon correlation function arise. In general, other graphs are needed for gauge invariance-see the discussion of Fig. 3 in the Appendix for more on this. Finally, the graphs contain no light cone divergences, so subtleties associated with Wilson lines and light cone regulators do not affect our calculations. (Of course, in more general higher-order graphs, all these issues will become important.) The result is a kind of spectator model that closely mirrors actual pQCD calculations.

Most of the steps needed to calculate each of the two terms on the right side of Eq. (11) can be found in already existing literature [42,62], with only slight modifications needed in the twist-3 quark-gluon correlation function case to convert to dimensional regularization and minimal subtraction. (See Appendix for a discussion of these calculations.) Model calculations of transverse-spin and momentum effects were also calculated earlier in [63-66]. While the complete result for $\Delta f_{1 T}^{\perp}\left(k_{c}\right)$ is not relevant to our discussion, a very important result is that it involves double logarithmic terms with the quark mass $m_{q}$ of the form

$$
\begin{aligned}
& \Delta f_{1 T}^{\perp}\left(k_{c} \sim \mu\right) \\
& \quad=-\frac{C_{F} N_{c}}{2 \pi} \alpha_{s}(\mu)^{2} x(1-x) \ln ^{2}\left(\frac{\mu}{(1-x) m_{q}}\right)+\cdots .
\end{aligned}
$$

The "..." refers to all other terms not involving double logarithms of the form $\ln ^{2}(\mu /$ mass $)$.

To see that this creates complications, consider Eq. (12) expanded through the first several orders,

$$
\begin{aligned}
M \Delta f_{1 T}^{\perp}\left(k_{c}\right)= & \sum_{i j} \mathcal{C}_{i j}^{(2)}\left(x / x^{\prime}, L, \alpha_{s}(\mu)\right) \otimes T_{j(g) / H}^{(0)}(x ; \mu)+\sum_{i j} \mathcal{C}_{i j}^{(1)}\left(x / x^{\prime}, L, \alpha_{s}(\mu)\right) \otimes T_{j(g) / H}^{(1)}(x ; \mu) \\
& +\sum_{i j, b} m_{b, i j} \mathcal{C}_{b, i j}^{(2)}\left(x / x^{\prime}, L, \alpha_{s}\left(k_{c}\right)\right) \otimes h_{b, j / H}^{(0)}(x ; \mu)+\sum_{i j, b} m_{b, i j} \mathcal{C}_{b, i j}^{(1)}\left(x / x^{\prime}, L, \alpha_{s}\left(k_{c}\right)\right) \otimes h_{b, j / H}^{(1)}(x ; \mu) \\
& + \text { h.o. }+O\left(\frac{\Lambda_{\mathrm{QCD}}^{2}}{k_{c}^{2}}\right),
\end{aligned}
$$

with the (...) superscripts denoting the order in perturbation theory. If Eq. (12) were true, then one of these terms must contain the double logarithm in Eq. (13). But

$$
T_{j(g) / H}^{(1), \perp}(x ; \mu)=T_{j(g) / H}^{(0), \perp}(x ; \mu)=0,
$$

because at least two gluons (a spectator and a final state interaction) are needed for the correlation function to be nonzero. So if Eq. (14) could accommodate Eq. (13), then the $\alpha_{s}^{2} \ln ^{2}\left(\mu /\left((1-x) m_{q}\right)\right)$ would have to appear in either the fourth or fifth lines. However, the order $\alpha_{s}^{0} h_{b, i / H}^{(0)}(x ; \mu)$ and the order $\alpha_{s}^{1} h_{b, i / H}^{(1)}(x ; \mu)$ can contain at most zero and one $\ln (\mu)$ factors, respectively. This means at least one power of $\ln \left(\mu /\left((1-x) m_{q}\right)\right)$ would have to be included inside $\mathcal{C}_{b, i j}^{(2)}$ or $\mathcal{C}_{b, i j}^{(1)}$. If this were done, however, it would violate the requirement that no logarithms other than the mass-independent Eq. (8) appear in the hard $\mathcal{C}$-coefficients. This shows that the factorization in Eq. (14), and therefore Eq. (12) generally, is invalid.

An equivalent and more direct way to state the above is simply to note that since the coupling only vanishes like $\alpha_{s}(\mu) \sim 1 / \ln (\mu)$ for $\mu \gg \Lambda_{\mathrm{QCD}}$, then the term in Eq. (13) undergoes no suppression at large $\mu$.

It should be understood that, since the correlation functions are strictly speaking nonperturbative, the mass scales like the $m_{q}$ in Eq. (13) represent more general nonperturbative structures. In some ways, therefore, a model 


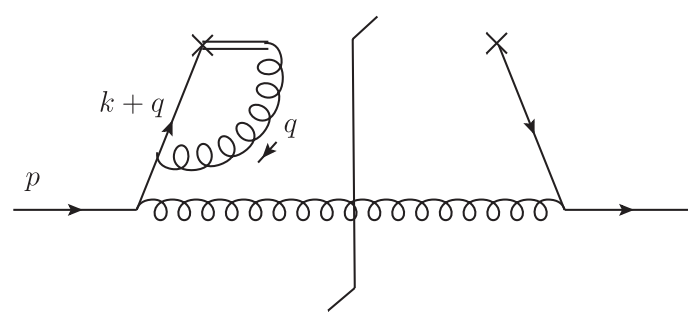

(a)

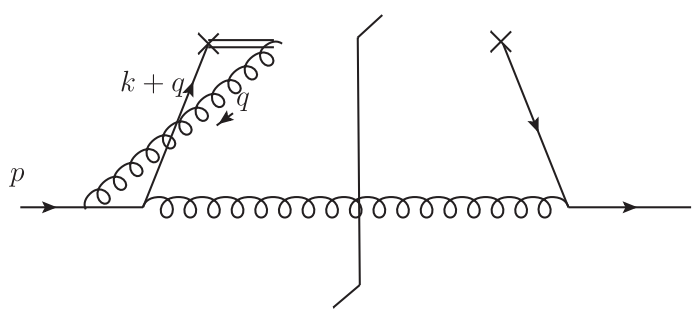

(b)

FIG. 3. Graphs (a) and (b), along with their Hermitian conjugates, are needed in general for gauge invariance. Analogous graphs are also needed for the collinear twist-3 function.

renormalizable diquark spectator theory is more illustrative of the problem described above, since mass scales like the $m_{q}$ in Eq. (13) become more complicated nonperturbative objects.

It is possibly tempting to argue that in a proton target terms like Eq. (13) would be suppressed by $m_{q} / M_{p}$ ratios. But this same ratio appears in all terms to all orders in the correlation function, so there is no relative suppression. This is especially clear in other model theories like a spectator diquark theory-see Eqs. (A7)-(A9). Thus, the double logarithm in Eq. (13) represents a kind of strong ultraviolet ambiguity that did not arise in the unpolarized case.

Furthermore, the fact that the double logarithm in Eq. (13) goes to infinity as the collinear regulator is removed, $m_{q} \rightarrow 0$, signals that the two sides of Eq. (4) have different collinear sensitivities (as $k_{T} \rightarrow 0$ ) manifested by the divergent $k_{T}$-integration starting from its $\mathrm{UV}$ perturbative region and using dimensional regularization. The need to account for this divergent $m_{q} \rightarrow 0$ behavior will reappear in the treatment of the very large transverse momentum $\left(Q_{T} \sim Q\right)$ region of physical processes like the Drell-Yan example in Sec. I.

Like in any QCD factorization approach to a physical observable, perturbative calculations of short-distance hard parts beyond the lowest-order tree level require perturbatively calculated and regularized partonic versions of the long-distance correlation functions to remove all soft and collinear divergences in the hard partonic scattering. Since the moment of the Sivers TMD function and the twist-3 correlation function in the two sides of Eq. (4) have different collinear sensitivities, the use of the long-distance correlation functions for QCD factorization treatment of weighted SSAs requires caution and needs to be made consistent with a factorization formalism.

\section{DISCUSSION}

The contribution to $\Delta f_{1 T}^{\perp}\left(k_{c}\right)$ in Eq. (13) makes clear that there is very strong sensitivity to choices in how the ultraviolet contribution to the integral over transverse momentum for weighted-integrated asymmetries is implemented. The two schemes we considered were (1) standard collinear renormalization for the twist-3 collinear correlation function and (2) direct integration of the TMD function (the Sivers function) with suppression of the large transverse momentum contribution. It is the latter method, however, that is almost always used in practical phenomenological applications. That is, parametrizations of the twist-3 collinear correlation function are usually constructed from the Sivers function via Eq. (4).

This implies that it is the evolution of the Sivers function, performed using standard TMD evolution techniques and before the integration over $k_{T}$, that governs the evolution of the weighted-integrated asymmetries as they are normally determined.

The technical reason for the term in Eq. (13) is that the box-loop integral in Fig. 2(a) produces a power of $\left(\mu /\left((1-x) m_{q}\right)^{2 \epsilon}\right.$ in dimensional regularization in addition to the $\left(\mu /\left((1-x) m_{q}\right)^{2 e}\right.$ that already comes from the divergent $k_{T}$ integral. In a calculation of the renormalized twist-3 function, both multiply a $1 / \epsilon^{2}$ from the divergent $k_{T}$-integral to produce two $\ln ^{2}\left(\mu /\left((1-x) m_{q}\right)\right)$ terms. By contrast, the TMD PDF calculation is finite at the order of graphs in Fig. 2, so $O(\epsilon)$ factors never contribute. The only relevant $\ln \left(\mu /\left((1-x) m_{q}\right)\right.$ in the integral of the TMD PDF comes directly from the cutoff transverse momentum integral when it is applied on the left side of Eq. (4). The result is that the $\ln ^{2}\left(\mu /\left((1-x) m_{q}\right)\right)$ term in the renormalized twist-3 correlation function comes with an extra factor of 2 compared with the $\ln ^{2}\left(\mu /\left((1-x) m_{q}\right)\right)$ term in the weighted integral of the Sivers function. Thus, the double logarithms like Eq. (13) do not cancel in Eq. (11).

The more general reason is that transverse momentum integrals do not commute with the removal of ultraviolet regulators, a property that has already been remarked upon in some detail in, for example, Ref. [42]. This results in a type of scale anomaly that already appears in the unpolarized leading twist case, Eq. (3). In Eq. (7), however, large contributions analogous to Eq. (13) do not arise because the transverse momentum integrals corresponding to the particular graphs in Fig. 2 are finite for the transverse momentum integral in Eq. (3).

Some physical intuition for the mismatch is gained by recalling that the design region for the TMD PDF treatment, where the approximations that give TMD factorization 
apply, is the very small $Q_{T} \ll Q$ region, including $Q_{T} \lesssim \Lambda_{\mathrm{QCD}}$, whereas the behavior of the TMD PDF at $k_{T}$ close to physical hard scales is not physically meaningful without some correction term. But the factor of $k_{T}^{2} / M^{2}$ in the integrand of Eq. (4) effectively discards the relevant $k_{T} \sim 0$ contribution to the cross section while amplifying the ill-defined contribution from $k_{T}>\mu$. Therefore, the resulting integral is dominated by an arbitrary scheme used to regulate the large $k_{T}$ behavior. In other words, TMD factorization derivations apply to cross sections differential in $Q_{T}$ and in the small $Q_{T}$ limit, but the $Q_{T}$ weighting suppresses this small $Q_{T}$ region (in fact creating a zero) while magnifying the $Q_{T} \sim Q$ region of the cross section where a different sort of factorization is needed. That the single $\mathbf{Q}_{T}$ weighting is the lowest power $\mathbf{Q}_{T}$ weight that gives a nonzero integrated transverse SSA does not mitigate the potential for such shifts in the important momentum range to spoil relations like Eq. (4).

The particular order in which transverse momentum integrals are evaluated and ultraviolet regulators are removed is important. In renormalized collinear correlation functions (like the twist-3 quark-gluon correlation function), the ultraviolet regulator needs to be the same for real and virtual emissions for ensuring such features as the automatic cancellations of light cone divergences in collinear correlation functions [67]. Thus, ultraviolet regulators can only be removed after all integrals are evaluated. By contrast, in the unintegrated TMD PDFs there are no regulators on real parton transverse momentum since the transverse momentum is fixed to values determined by the physical cross section. It is only at later stages that a $k_{T}^{2}$-weighted integral of a phenomenologically extracted Sivers function is performed, as in Eq. (4), at which point a cutoff on the physical region of $k_{T} \gtrsim \mu$ is restored in a separate step. This reversal in the natural order of regulator removal between the two cases is the origin of the problem discussed in the previous section.

Forcing a version of Eq. (4) amounts to dealing with issues such as light cone divergences in the twist-3 quark-gluon correlation function point-by-point in parton transverse momentum first, before transverse momentum integrals with real emissions are evaluated. This allows separate ultraviolet regulators to be applied to real and virtual ultraviolet divergences. Then it is possible to impose the requirement that the weighted Sivers and twist-3 calculations use the same ultraviolet regulators on real emissions from the outset, thus ensuring Eq. (4). This is equivalent to defining the TMD PDF first and then defining the corresponding twist-3 function via the weighted transverse momentum integral of the TMD function. In this view, Eq. (4) should be viewed as a definition rather than a derived result. Nevertheless, such a convention preserves the logical structure embodied in relations like Eq. (4) and thereby allows twist-3 calculations an interpretation in terms of intrinsic transverse momentum. ${ }^{2}$ This then provides one answer to the question of which type of scale evolution is relevant in weighted integrals of spin asymmetries, in cases where large transverse momentum is strongly suppressed. If, as we suggest above, the collinear $T_{q(g) / H}(x)$ on the right side of Eq. (4) is defined via the TMD PDF on the left side, then evolution is dictated by the TMD evolution of $f_{1 T, q / H}^{\perp}\left(x, k_{\mathrm{T}}\right)$ at small transverse momentum. Of course, at very large $Q$ the integral becomes dominated by nonintrinsic perturbatively generated transverse momentum radiation [68], and a switch to a scheme like [26] may then be useful to exploit refactorization.

Obtaining a fully fixed prescription for treating divergences in parton correlation functions requires complete factorization treatments for specific processes, to clarify how those parton correlation functions contribute to the evaluation of corresponding hard parts. We emphasize that more work in this direction is needed.

A potential complication is that if the twist-3 function is defined via TMD PDFs, then it might inherit some of the problems with TMD factorization that can arise in hadronhadron collisions with measured hadron transverse momentum in the final state [69,70]. Such effects may be mitigated, however, if scales are evolved high enough that the integrand is dominated by a perturbatively generated tail. Moreover, a full treatment of the matching to the large $Q_{T} \sim Q$ region is needed. We leave the investigation of all such issues to future work.

\section{ACKNOWLEDGMENTS}

We thank F. Aslan, L. Gamberg, A. Metz, and D. Pitonyak for helpful discussion. This work is supported in part by the U.S. Department of Energy Award No. DE-AC0506OR23177, under which Jefferson Science Associates, LLC, manages and operates Jefferson Lab. T. R. was also supported by the U.S. Department of Energy, Office of Science, Office of Nuclear Physics, under Award No. DESC0018106. B. W. is supported in part by the National Science Foundation of China (11875232) and the Zhejiang University Fundamental Research Funds for the Central Universities. This work is also supported in part within the framework of the TMD Topical Collaboration.

\section{APPENDIX: CALCULATION OF $\Delta f_{1 T}^{\perp}\left(\boldsymbol{k}_{c}\right)$}

Here we explain some of the details leading to Eq. (13). Since the basic integrals have all been set up before [42], we will simply refer to earlier literature, only modifying those parts needed to implement renormalization with dimensional regularization and minimal subtraction.

\footnotetext{
${ }^{2}$ Note that results like [26] amount only to one of potentially many arbitrary regulator schemes for the integral on the left side of Eq. (4) and are not actual derivations of Eq. (4). Specifically, they do not address the question of regulator sensitivity.
} 


\section{The scalar field spectator}

It will be simplest to structure the argument by starting with the result for the scalar diquark model and explain the steps to transform to QCD. We start from Eq. (29) in Ref. $[42]^{3}$ in the scalar model. Here we adopt a sign convention consistent with Eq. (9) and compute the integrals over $\boldsymbol{k}_{\mathrm{T}}$ and $\boldsymbol{q}_{\mathrm{T}}$ in $n=2-2 \epsilon$ dimensions. In dimensional regularization, the only $n$ dependence is from the integration measure and the factor $\mu^{4 \epsilon}$ that comes with the couplings. With the pointlike coupling between the nucleon, quark, and spectator diquark, the integral in that equation becomes, up to overall factors,

$$
\begin{aligned}
I_{T} \equiv & \int \frac{\mathrm{d}^{n} \boldsymbol{k}_{\mathrm{T}}}{(2 \pi)^{n}} \frac{\mathrm{d}^{n} \boldsymbol{q}_{\mathrm{T}}}{(2 \pi)^{n}} \frac{q_{\mathrm{T}}^{2}-\left(\boldsymbol{q}_{\mathrm{T}} \cdot \boldsymbol{S}_{\mathrm{T}}\right)^{2}}{q_{\mathrm{T}}^{2}\left[k_{\mathrm{T}}^{2}+\Lambda_{s}^{2}\right]\left[\left(\boldsymbol{k}_{\mathrm{T}}+\boldsymbol{q}_{\mathrm{T}}\right)^{2}+\Lambda_{s}^{2}\right]} \\
= & \frac{\pi^{n / 2} \Gamma(2-n / 2)}{\Gamma(2)} \int_{0}^{1} \mathrm{~d} \alpha \int \frac{\mathrm{d}^{n} \boldsymbol{q}_{\mathrm{T}}}{(2 \pi)^{2 n}} \frac{1}{q_{\mathrm{T}}^{2}} \\
& \times\left[q_{\mathrm{T}}^{2}-\left(\boldsymbol{q}_{\mathrm{T}} \cdot \boldsymbol{S}_{\mathrm{T}}\right)^{2}\right]\left[\alpha(1-\alpha) q_{\mathrm{T}}^{2}+\Lambda_{s}^{2}\right]^{n / 2-2},
\end{aligned}
$$

where

$$
\Lambda_{s}^{2}=x M_{s}^{2}+(1-x) m_{q}^{2}-x(1-x) M^{2},
$$

with $M_{s}, m_{q}$, and $M$ being the masses of the scalar diquark, quark, and nucleon, respectively. By choosing the orientations

$$
\begin{gathered}
\boldsymbol{q}_{\mathrm{T}}=q_{\mathrm{T}}\left(\sin \theta_{1} \sin \theta_{2} \cdots \sin \theta_{n-1}, \ldots, \cos \theta_{1}\right), \\
\boldsymbol{S}_{\mathrm{T}}=(0, \ldots, 1)
\end{gathered}
$$

in $n$ dimensions [Eq. (A3) takes the form of the standard $n$-dimensional spherical coordinates, while in Eq. (A4) all components of $\boldsymbol{S}_{\mathrm{T}}$ are zero except for the last one], it is straightforward to carry out the angular part of the integral and verify

$$
\int \mathrm{d}^{n} \boldsymbol{q}_{\mathrm{T}} q_{\mathrm{T}}^{2}=n \int \mathrm{d}^{n} \boldsymbol{q}_{\mathrm{T}}\left(\boldsymbol{q}_{\mathrm{T}} \cdot \boldsymbol{S}_{\mathrm{T}}\right)^{2}
$$

Then it is valid in Eq. (A1) to replace $q_{\mathrm{T}}^{2}-\left(\boldsymbol{q}_{\mathrm{T}} \cdot \boldsymbol{S}_{\mathrm{T}}\right)^{2} \rightarrow$ $(1-1 / n) q_{\mathrm{T}}^{2}$ and obtain

$$
\begin{aligned}
I_{T} & =\frac{\pi^{n / 2} \Gamma(2-n / 2)}{\Gamma(2)}\left(1-\frac{1}{n}\right) \int_{0}^{1} \mathrm{~d} \alpha \int \frac{\mathrm{d}^{n} \boldsymbol{q}_{\mathrm{T}}}{(2 \pi)^{n}}\left[\alpha(1-\alpha) q_{\mathrm{T}}^{2}+\Lambda_{s}^{2}\right]^{n / 2-2} \\
& =\frac{\pi^{n}}{(2 \pi)^{2 n}}\left(1-\frac{1}{n}\right) \Gamma(2-n) \Lambda_{s}^{2(n-2)} \int_{0}^{1} \mathrm{~d} \alpha[\alpha(1-\alpha)]^{-n / 2} \\
& =\frac{\pi^{n}}{(2 \pi)^{2 n}}\left(1-\frac{1}{n}\right) \Gamma(2-n) \Lambda_{s}^{2(n-2)} \frac{\Gamma^{2}(\epsilon)}{\Gamma(2 \epsilon)} .
\end{aligned}
$$

Restoring the overall factors dropped in Eq. (A1) and expanding near $\epsilon=0$ gives the logarithmic terms with

$$
-\frac{1}{M} T_{q(g) / H}(x ; \mu)=\frac{N_{c} C_{F} g \lambda_{s}^{2} g_{s}}{16 \pi^{3}}(1-x)\left(\frac{m_{q}}{M}+x\right)\left(\ln ^{2}\left(\Lambda_{s} / \mu\right)+\frac{1}{2}\left(1+2 \gamma_{E}-2 \ln (4 \pi)\right) \ln \left(\Lambda_{s} / \mu\right)\right)+\cdots .
$$

The analog of Eq. (A6) for the weighted Sivers function comes from the integral in Eq. (49) in Ref. [42], which was also calculated in [62]. Without overall factors the integral is

$$
I_{S} \equiv \frac{1}{4 \pi M^{2}} \int_{0}^{k_{c}} \frac{\mathrm{d} k_{\mathrm{T}}}{2 \pi} \frac{k_{\mathrm{T}}}{k_{\mathrm{T}}^{2}+\Lambda_{s}^{2}} \ln \frac{k_{\mathrm{T}}^{2}+\Lambda_{s}^{2}}{\Lambda_{s}^{2}}=\frac{1}{32 \pi^{2} M^{2}} \ln ^{2}\left(k_{c}^{2} / \Lambda_{s}^{2}+1\right)
$$

Cutting off the $k_{\mathrm{T}}$ integral at $k_{c}=\mu$, restoring the overall factors dropped in Eq. (A8), and expanding to lowest order in $\Lambda_{s} / \mu$,

$$
\int \mathrm{d}^{2} \boldsymbol{k}_{\mathrm{T}} \frac{k_{\mathrm{T}}^{2}}{M^{2}} f_{1 T}^{\perp}\left(x, k_{\mathrm{T}}\right)=\frac{N_{c} C_{F} g \lambda_{s}^{2} g_{s}}{32 \pi^{3}}(1-x)\left(\frac{m_{q}}{M}+x\right) \ln ^{2}\left(\Lambda_{s} / \mu\right)+O\left(\frac{\Lambda_{\mathrm{QCD}}^{2}}{\mu^{2}}\right) .
$$

Note the factor of 2 difference between the double logarithmic terms in Eqs. (A9) and (A7). Subtracting Eqs. (A9) and (A7) gives a version of Eq. (13) for the case of a scalar field for the spectator.

\footnotetext{
${ }^{3}$ Equation (44) of Ref. [42] differs by a sign from Eq. (9) due to a different convention for the direction of the Wilson line. There it is chosen to be consistent with Drell-Yan-like processes.
} 


\section{QCD}

Transitioning to the case of QCD with an incoming quark of mass $m_{q}$ primarily entails a change in the Dirac trace. The trace part of the Sivers calculation in the diquark model is

$$
\begin{aligned}
\operatorname{Tr}_{\mathrm{S}}^{\text {diquark }} & \equiv \operatorname{Tr}\left[\gamma^{+}\left(\not k+\not l+m_{q}\right)(\not p+M) \gamma^{5} \$_{T}\left(\not k+m_{q}\right)\right](2 p-2 k-q)^{\tau} n_{\tau} \\
& =8 i p^{+2}(1-x)\left(M x+m_{q}\right) \epsilon^{i j} q_{T i} s_{T j},
\end{aligned}
$$

where the $i \pi \delta\left(q^{+}\right)$from the eikonal propagator of the Wilson line constrains $q^{+}=0$ and gives the imaginary part of the $q$ integral [see Eq. (94) of Ref. [62] ]. In QCD this trace becomes

$$
\begin{aligned}
\operatorname{Tr}_{\mathrm{S}}^{\mathrm{QCD}} \equiv & \operatorname{Tr}\left[\gamma^{+}\left(\not k+\not k+m_{q}\right) \gamma^{\mu}\left(\not p+m_{q}\right) \gamma^{5} \phi_{T} \gamma^{\alpha}\left(\not k+m_{q}\right)\right] d_{\mu \nu}(p-k-q) d_{\sigma \alpha}(p-k) \times\left[(2 p-2 k-q)^{\tau} g^{\nu \sigma}\right. \\
& \left.+(k-p-q)^{\nu} g^{\sigma \tau}+(2 q-p+k)^{\sigma} g^{\tau \nu}\right] n_{\tau} \\
= & 16 i p^{+2} x m_{q}(x-1) \epsilon^{i j} q_{\mathrm{Ti}} s_{T j},
\end{aligned}
$$

where we work in Feynman gauge with the gluon polarization tensor (no ghost graphs contribute at this order):

$$
d_{\mu \nu}(q)=-g_{\mu \nu}
$$

Similarly, the traces for the twist-3 quark-gluon correlation function in the two theories are

$$
\begin{gathered}
\operatorname{Tr}_{\mathrm{T}}^{\text {diquark }} \equiv \operatorname{Tr}\left[\gamma^{+}\left(\not k+\not h+m_{q}\right)(\not p+M) \gamma^{5} \phi_{T}\left(\not k+m_{q}\right)\right] \epsilon^{s_{T} \rho n \bar{n}}(2 p-2 k-q)^{\tau}\left(-n_{\tau} q_{\rho}\right) \\
=-8 i p^{+2}(1-x)\left(M x+m_{q}\right)\left[q_{\mathrm{T}}^{2}-\left(\boldsymbol{q}_{\mathrm{T}} \cdot \boldsymbol{S}_{\mathrm{T}}\right)^{2}\right] \\
\operatorname{Tr}_{\mathrm{T}}^{\mathrm{QCD}} \equiv \operatorname{Tr}\left[\gamma^{+}\left(\not k+\not k+m_{q}\right) \gamma^{\mu}\left(\not p+m_{q}\right) \gamma^{5} \oiint_{T} \gamma^{\alpha}\left(\not k+m_{q}\right)\right] d_{\mu \nu}(p-k-q) d_{\sigma \alpha}(p-k)\left[(2 p-2 k-q)^{\tau} g^{\nu \sigma}+(k-p-q)^{\nu} g^{\sigma \tau}\right. \\
\left.+(2 q-p+k)^{\sigma} g^{\tau \nu}\right] \epsilon^{s_{T} \rho n \bar{n}}\left(-n_{\tau} q_{\rho}\right) \\
=-8 i p^{+2}(n x+2-n) m_{q}(x-1)\left[q_{\mathrm{T}}^{2}-\left(\boldsymbol{q}_{\mathrm{T}} \cdot \boldsymbol{S}_{\mathrm{T}}\right)^{2}\right] .
\end{gathered}
$$

The trace part of the twist-3 quark-gluon correlation function in QCD acquires a dependence on the dimension $n$. Note that for Sivers function we always work in four dimensions $(n=2)$, so $\operatorname{Tr}_{\mathrm{S}}^{\mathrm{QCD}}$ does not have a similar factor. In the case of twist- 3 correlation function, $q^{+}=0$ is imposed by the delta function from the cut vertex [38,42]. The $n$ and $\bar{n}$ four-vectors in the above traces represent lightlike vectors in the minus and plus directions, respectively, not to be confused with the spacetime dimension.

In general, the graphs in Fig. 3 are also needed to give gauge invariance, but we have confirmed that they do not contribute in either the twist-3 collinear or TMD calculations when the target is transversely polarized and the active quark is unpolarized.

The momentum integrals in QCD and diquark model are almost identical for both Sivers and collinear twist-3 functions, with only the replacement

$$
\Lambda_{s}^{2} \rightarrow \Lambda_{g}^{2} \equiv(1-x)^{2} m_{q}^{2} .
$$

Also note that in the transition to QCD the change of the coupling constants and the color factors are the same for Sivers and twist-3 PDFs,

$$
g g_{s} \lambda_{s}^{2} \rightarrow 16 \pi^{2} \alpha_{S}^{2}, \quad N_{c} C_{F} \rightarrow-\frac{1}{2} N_{c} C_{F} .
$$

Aside from the above replacements, for Sivers in QCD one needs $(1-x)\left(M x+m_{q}\right) \rightarrow 2 m_{q} x(x-1)$, as can be seen from Eqs. (A10) and (A11). The resulting logarithmic terms are

$$
L_{S}=\frac{N_{c} C_{F} \alpha_{S}^{2}}{2 \pi} x(1-x) \ln ^{2}\left(\Lambda_{g} / \mu\right) .
$$

For the twist-3 quark-gluon correlation function, however, we must change $(1-x)\left(M x+m_{q}\right) \rightarrow m_{q}(n x+2-n)(x-1)$ and include the factor $(n x+2-n)$ when expanding near $\epsilon=0$ if we wish to obtain all logarithms beyond the double logarithm. The result is

$$
\begin{aligned}
L_{T}= & -\frac{N_{c} C_{F} \alpha_{S}^{2}}{\pi} x(1-x)\left(\ln ^{2}\left(\Lambda_{g} / \mu\right)\right. \\
& \left.+\left(1+\gamma_{E}-\ln (4 \pi)-\frac{1}{2 x}\right) \ln \left(\Lambda_{g} / \mu\right)\right) .
\end{aligned}
$$

From Eq. (11)

$$
\begin{aligned}
& \Delta f_{1 T}^{\perp}\left(k_{c}\right)=L_{T}+L_{S}+\cdots \\
& \quad=-\frac{N_{c} C_{F} \alpha_{S}^{2}}{2 \pi} x(1-x) \ln ^{2}\left(\Lambda_{g} / \mu\right)+\cdots .
\end{aligned}
$$


[1] G. Bunce et al., Phys. Rev. Lett. 36, 1113 (1976).

[2] D. L. Adams et al. (E581, E704 Collaborations), Phys. Lett. B 261, 201 (1991).

[3] A. Bravar et al. (Fermilab E704 Collaboration), Phys. Rev. Lett. 77, 2626 (1996).

[4] C. A. Aidala, S. D. Bass, D. Hasch, and G. K. Mallot, Rev. Mod. Phys. 85, 655 (2013).

[5] G. L. Kane, J. Pumplin, and W. Repko, Phys. Rev. Lett. 41, 1689 (1978).

[6] A. Accardi et al., Eur. Phys. J. A 52, 268 (2016).

[7] J. C. Collins, D. E. Soper, and G. F. Sterman, Adv. Ser. Dir. High Energy Phys. 5, 1 (1989).

[8] D. W. Sivers, Phys. Rev. D 41, 83 (1990).

[9] D. W. Sivers, Phys. Rev. D 43, 261 (1991).

[10] S. J. Brodsky, D. S. Hwang, and I. Schmidt, Nucl. Phys. B642, 344 (2002).

[11] A. V. Efremov and O. V. Teryaev, Phys. Lett. 150B, 383 (1985).

[12] J.-W. Qiu and G. F. Sterman, Phys. Rev. Lett. 67, 2264 (1991).

[13] J.-W. Qiu and G. F. Sterman, Nucl. Phys. B378, 52 (1992).

[14] J.-W. Qiu and G. F. Sterman, Phys. Rev. D 59, 014004 (1998).

[15] J. C. Collins, D. E. Soper, and G. Sterman, Nucl. Phys. B250, 199 (1985).

[16] J. Collins, Cambridge Monogr. Part. Phys., Nucl. Phys., Cosmol. 32, 1 (2011).

[17] X.-D. Ji, J.-P. Ma, and F. Yuan, Phys. Rev. D 71, 034005 (2005).

[18] X.-D. Ji, J.-P. Ma, and F. Yuan, Phys. Lett. B 597, 299 (2004).

[19] J.-W. Qiu and G. Sterman, Nucl. Phys. B353, 137 (1991).

[20] J.-W. Qiu and G. F. Sterman, AIP Conf. Proc. 223, 249 (1991).

[21] X. Ji, J.-W. Qiu, W. Vogelsang, and F. Yuan, Phys. Rev. Lett. 97, 082002 (2006).

[22] X. Ji, J.-W. Qiu, W. Vogelsang, and F. Yuan, Phys. Rev. D 73, 094017 (2006).

[23] I. Scimemi, A. Tarasov, and A. Vladimirov, J. High Energy Phys. 05 (2019) 125.

[24] Z.-B. Kang, I. Vitev, and H. Xing, Phys. Rev. D 87, 034024 (2013).

[25] L.-Y. Dai, Z.-B. Kang, A. Prokudin, and I. Vitev, Phys. Rev. D 92, 114024 (2015).

[26] L. Gamberg, A. Metz, D. Pitonyak, and A. Prokudin, Phys. Lett. B 781, 443 (2018).

[27] H. Xing and S. Yoshida, Adv. High Energy Phys. 2019, 4825790 (2019).

[28] X. Luo and H. Sun, Phys. Rev. D 101, 074016 (2020).

[29] E. L. Berger and J.-W. Qiu, Phys. Rev. Lett. 91, 222003 (2003).

[30] D. Boer, P. J. Mulders, and F. Pijlman, Nucl. Phys. B667, 201 (2003).

[31] Z.-B. Kang, J.-W. Qiu, W. Vogelsang, and F. Yuan, Phys. Rev. D 83, 094001 (2011).

[32] Z.-B. Kang and A. Prokudin, Phys. Rev. D 85, 074008 (2012).

[33] A. Metz, D. Pitonyak, A. Schfer, M. Schlegel, W. Vogelsang, and J. Zhou, Few Body Syst. 56, 331 (2015).

[34] L. Gamberg, Z.-B. Kang, D. Pitonyak, and A. Prokudin, Phys. Lett. B 770, 242 (2017).

[35] K. Kanazawa, Y. Koike, A. Metz, and D. Pitonyak, Phys. Rev. D 89, 111501 (2014).
[36] L. Gamberg and Z.-B. Kang, Phys. Lett. B 696, 109 (2011).

[37] M. G. Alexeev et al. (COMPASS Collaboration), Nucl. Phys. B940, 34 (2019).

[38] Z.-B. Kang and J.-W. Qiu, Phys. Rev. D 79, 016003 (2009).

[39] V. Braun, A. Manashov, and B. Pirnay, Phys. Rev. D 80, 114002 (2009); 86, 119902(E) (2012).

[40] J. C. Collins and D. E. Soper, Nucl. Phys. B197, 446 (1982).

[41] S. M. Aybat, J. C. Collins, J.-W. Qiu, and T. C. Rogers, Phys. Rev. D 85, 034043 (2012).

[42] Z.-B. Kang, J.-W. Qiu, and H. Zhang, Phys. Rev. D 81, 114030 (2010).

[43] M. Anselmino, M. Boglione, and S. Melis, Phys. Rev. D 86, 014028 (2012).

[44] M. Boglione, U. D’Alesio, C. Flore, and J. O. GonzalezHernandez, J. High Energy Phys. 07 (2018) 148.

[45] J. Cammarota, L. Gamberg, Z.-B. Kang, J. A. Miller, D. Pitonyak, A. Prokudin, T. C. Rogers, and N. Sato, arXiv: 2002.08384.

[46] A. Metz and D. Pitonyak, Phys. Lett. B 723, 365 (2013); 762, 549(E) (2016).

[47] F. Yuan and J. Zhou, Phys. Rev. Lett. 103, 052001 (2009).

[48] Z.-B. Kang, A. Prokudin, P. Sun, and F. Yuan, Phys. Rev. D 93, 014009 (2016).

[49] P. J. Mulders and R. D. Tangerman, Nucl. Phys. B461, 197 (1996); B484, 538(E) (1997).

[50] J. C. Collins and D. E. Soper, Nucl. Phys. B194, 445 (1982).

[51] M. Diehl, Eur. Phys. J. A 52, 149 (2016).

[52] T. C. Rogers, Eur. Phys. J. A 52, 153 (2016).

[53] I. W. Stewart, F. J. Tackmann, and W. J. Waalewijn, Phys. Rev. D 81, 094035 (2010).

[54] T. Becher and M. Neubert, Eur. Phys. J. C 71, 1665 (2011).

[55] T. Becher, M. Neubert, and D. Wilhelm, J. High Energy Phys. 02 (2012) 124.

[56] T. Becher, M. Neubert, and D. Wilhelm, J. High Energy Phys. 05 (2013) 110.

[57] M. G. Echevarría, A. Idilbi, and I. Scimemi, J. High Energy Phys. 07 (2012) 002.

[58] M. G. Echevarria, A. Idilbi, and I. Scimemi, Phys. Lett. B 726, 795 (2013).

[59] M. G. Echevarria, A. Idilbi, and I. Scimemi, Phys. Rev. D 90, 014003 (2014).

[60] J.-Y. Chiu, A. Jain, D. Neill, and I. Z. Rothstein, J. High Energy Phys. 05 (2012) 084.

[61] Y. Li, D. Neill, and H. X. Zhu, arXiv:1604.00392.

[62] A. Bacchetta, F. Conti, and M. Radici, Phys. Rev. D 78, 074010 (2008).

[63] X.-D. Ji and F. Yuan, Phys. Lett. B 543, 66 (2002).

[64] L. P. Gamberg, G. R. Goldstein, and K. A. Oganessyan, Phys. Rev. D 67, 071504 (2003).

[65] K. Goeke, S. Meissner, A. Metz, and M. Schlegel, Phys. Lett. B 637, 241 (2006).

[66] L. P. Gamberg, G. R. Goldstein, and M. Schlegel, Phys. Rev. D 77, 094016 (2008).

[67] J. C. Collins, Acta Phys. Pol. B 34, 3103 (2003).

[68] M. Grewal, Z.-B. Kang, J.-W. Qiu, and A. Signori, arXiv: 2003.07453.

[69] J. Collins and J.-W. Qiu, Phys. Rev. D 75, 114014 (2007).

[70] T. C. Rogers and P. J. Mulders, Phys. Rev. D 81, 094006 (2010). 